\title{
Plasma and Cerebrospinal Fluid Pharmacokinetics of Moxifloxacin in a Patient with Tuberculous Meningitis $\nabla$
}

The first line of treatment for tuberculosis (TB) consists of a combination of isoniazid (INH), rifampin (RIF), pyrazinamide (PZA), and ethambutol (EMB). In case of adverse events or resistance to one of these first-line drugs, fluoroquinolones are the primary treatment option (1). In vitro data suggest that moxifloxacin (MFX) is one of the most active compounds among fluoroquinolones (6). In vivo studies in murine models of TB have improved our understanding of optimal MFX dosing (14). In a randomized controlled trial in which MFX or EMB was added to a standard regimen of INH, RIF, and PZA, patients on MFX had their sputum converted slightly earlier to smear negative than did EMB-treated subjects, while adverse effects were similar (2). Several studies show that long-term MFX treatment at a dose of $400 \mathrm{mg}$ is well tolerated $(3,11)$, but safety data of higher dosages are lacking and have not been explored in clinical trials.

Overall, pharmacokinetic (PK) data on the optimal treatment of TB patients with MFX are limited. To our knowledge, data on cerebrospinal fluid (CSF) penetration in humans have not been published. In animals, data on MFX penetration of CSF show a CSF/plasma ratio of 23 to $50 \%$ in the absence of meningitis and 50 to $78 \%$ in active meningitis $(5,7)$. Drug interactions may also further complicate the PK of MFX (4, 13). We therefore decided to monitor plasma and CSF MFX concentrations over time to optimize treatment in a patient with intracerebral and leptomeningeal involvement of TB without active meningitis who could not tolerate INH.

After 2 months of ambulatory treatment, a 31-year-old man was admitted to the Beatrixoord Center for Tuberculosis because of excessive weight loss $(>25 \mathrm{~kg})$ and clinical deterioration. He was diagnosed as having miliary $\mathrm{TB}$ with central nervous involvement, including nonactive tuberculous meningitis, cerebral tuberculoma, and cervical spinal cord tuberculoma, which was confirmed by CSF testing and magnetic resonance imaging investigation. The ambulatory treatment comprised oral treatment with INH (300 mg), RIF (600 mg), EMB (1,200 mg), and PZA (1,500 mg) in a once-daily regimen. $\mathrm{He}$ also received prednisolone (15 $\mathrm{mg}$ twice daily) and omeprazole $(40 \mathrm{mg})$. One month after the start of the ambulatory treatment, the patient developed axonal polyneuropathy, which was characterized by an impaired walking pattern. INH toxicity was suspected; hence, INH was stopped and changed to oral MFX (400 mg once daily). The switch to MFX was made 3 months after diagnosis. The patient was transferred to the Neurology Department of the University Medical Center Groningen for pharmacologic assessment of the CSF penetration of MFX at different dosages. At this time point, the CSF was clear and had a normal color. It contained a total protein value of $0.69 \mathrm{~g} /$ liter, a glucose level of $2.2 \mathrm{~mol} / \mathrm{liter}$, and a mononuclear cell count of $19 \times 10^{6} /$ liter. At admission, his body temperature was 38 to $39^{\circ} \mathrm{C}$. In the month after stopping INH and starting MFX, the patient's overall clinical status improved and his walking impairment partly resolved.

A therapeutic drug-monitoring protocol was designed to assess the PK parameters of MFX in this patient with intracerebral and leptomeningeal TB. Steady-state plasma and CSF concentrations of MFX, given once daily in oral doses of 400 and $800 \mathrm{mg}$, were measured at day 3 after the administration of each dose. The MFX concentrations in CSF after the administration of two doses (400 and $800 \mathrm{mg}$ ) were explored to assess the effect of a dosage increase on the drug concentration in CSF. This could be necessary if the MFX concentration in CSF at a dosage of $400 \mathrm{mg}$ were too low.

An external lumbar drain and a peripheral intravenous catheter were inserted and used for PK sampling. The designed PK sampling procedure was limited to 1 week, as this was the maximum period for the external lumbar drain to be maintained in place.

Patency of the peripheral catheter was maintained by a saline drip. Before the PK sample was taken, the drip was stopped and the first $4 \mathrm{ml}$ of blood was discarded. To assess the safety of MFX at a dosage higher than that advised in the product leaflet, liver and renal function tests were performed. Electrocardiogram (ECG) monitoring was performed once a week during treatment to assess possible QTc prolongation. As the nature of this protocol was patient care, the local ethics committee approved the treatment protocol and informed consent was obtained.

Blood and CSF samples were collected into heparin-containing tubes before MFX administration and at 2, 4, 8, 12, and $24 \mathrm{~h}$ after dose administration. Plasma was separated by centrifugation at $1,500 \times g$ for $10 \mathrm{~min}$ at room temperature, transferred into propylene vials, and stored at $-20^{\circ} \mathrm{C}$ until analysis. Samples were analyzed by a validated high-performance liquid chromatography method with fluorescence detection (9). PK parameters were assessed with the KINFIT module of MWPharm (Mediware, The Netherlands). The patient's isolate was subjected to laboratory diagnosis at the Dutch National Mycobacteria Reference Laboratory (National Institute for Public Health and the Environment, RIVM). The Middlebrook 7H10 agar dilution method was applied for drug susceptibility testing of the patient's isolate(s) (12). The area under the concentration-time curve from 0 to $24 \mathrm{~h}\left(\mathrm{AUC}_{0-24}\right) /$ MIC ratio was calculated to estimate the adequate dose for treatment continuation (8). The choice of this ratio instead of maximum drug concentration in serum $\left(C_{\max }\right)$ ratios was de-

TABLE 1. MFX PK data

\begin{tabular}{|c|c|c|c|c|}
\hline \multirow{2}{*}{ Parameter $^{a}$} & \multicolumn{2}{|c|}{ Plasma } & \multicolumn{2}{|c|}{$\mathrm{CSF}$} \\
\hline & $400 \mathrm{mg}$ & $800 \mathrm{mg}$ & $400 \mathrm{mg}$ & $800 \mathrm{mg}$ \\
\hline $\mathrm{AUC}_{0-24}(\mathrm{mg} \cdot \mathrm{h} /$ liter $)$ & 8.9 & 19.4 & 8.4 & 14.3 \\
\hline Free $\mathrm{AUC}_{0-24}{ }^{b}(\mathrm{mg} \cdot \mathrm{h} /$ liter $)$ & 5.3 & 11.6 & 8.4 & 14.3 \\
\hline$C_{\max }(\mathrm{mg} /$ liter $)$ & 1.06 & 2.45 & 0.616 & 1.42 \\
\hline$T_{\max }(\mathrm{h})$ & 2 & 2 & 4 & 4 \\
\hline$C_{\min }(\mathrm{mg} /$ liter $)$ & 0.07 & 0.05 & 0.03 & 0.06 \\
\hline$t_{1 / 2}(\mathrm{~h})$ & 4.55 & 4.09 & 5.52 & 5.20 \\
\hline $\mathrm{CL} / \mathrm{F}$ & 71.34 & 32.3 & 81.1 & 46.0 \\
\hline $\mathrm{AUC}_{0-24} / \mathrm{MIC}$ ratio & 59.3 & 129.3 & 56.0 & 95.3 \\
\hline Free $\mathrm{AUC}_{0-24} / \mathrm{MIC}$ ratio & 35.3 & 77.3 & 56.0 & 95.3 \\
\hline
\end{tabular}

${ }^{a}$ Abbreviations: $C_{\max }$, maximum concentration of drug in plasma or CSF; $T_{\max }$, time to maximum concentration of drug in serum; $C_{\min }$, minimum concentration of drug in plasma or CSF; $t^{1 / 2}$, half-life; CL/F, apparent total body clearance.

${ }^{b}$ Calculated with a protein binding of $40 \%$. 


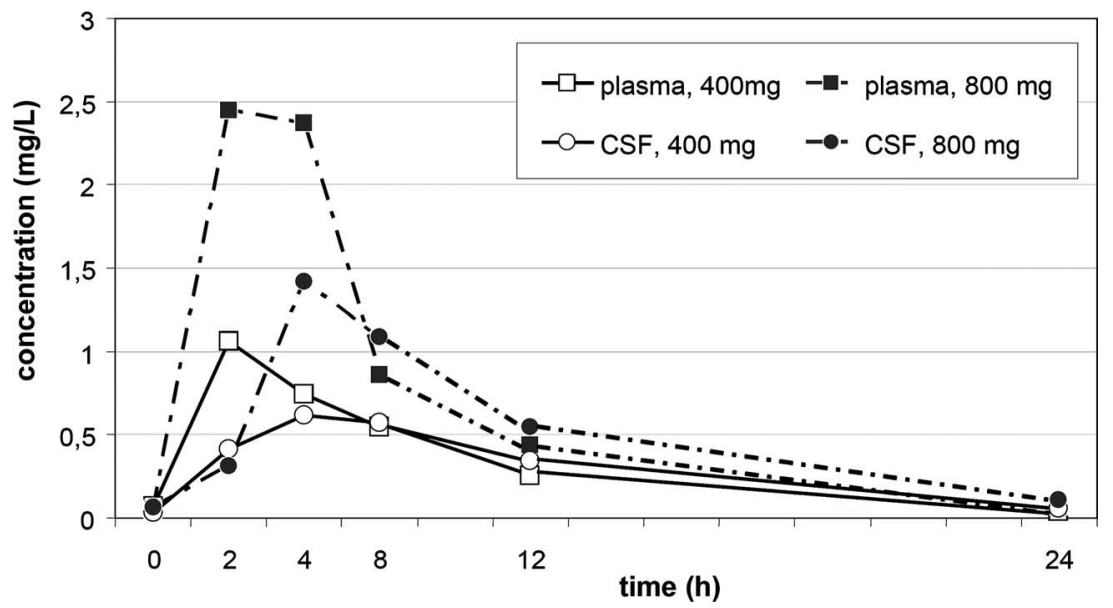

FIG. 1. MFX concentration-time curves in plasma and CSF at different drug dosages during steady state.

rived from an aerosol infection model of TB in BALB/c mice (8). This study concluded that the AUC/MIC ratio was the best $\mathrm{PK} /$ pharmacodynamic parameter to describe the in vivo efficacy of MFX against Mycobacterium tuberculosis.

PK parameters are shown in Table 1 . The MFX concentration-time curve is shown in Fig. 1. Drug susceptibility testing of M. tuberculosis showed an MFX MIC of $0.150 \mathrm{mg} /$ liter. The $\mathrm{AUC}_{\mathrm{CSF}} / \mathrm{AUC}_{\text {plasma }}$ ratio was 0.94 at $400 \mathrm{mg}$ of $\mathrm{MFX}$ once daily and 0.74 at $800 \mathrm{mg}$ of MFX once daily. The safety parameters assessed were ECG and blood sampling of renal and liver function. ECG monitoring showed a mean QTc of $432 \mathrm{~ms}$ (range, 431 to $432 \mathrm{~ms}$ ) during treatment with $400 \mathrm{mg}$ once daily, while once daily 800-mg treatment showed a mean QTc of $442 \mathrm{~ms}$ (range, 439 to $447 \mathrm{~ms}$ ). Renal and liver function tests remained within the normal range during MFX treatment. No side effects were reported that could be attributed to MFX.

This is the first report of measurements of combined CSF and plasma MFX concentrations over time in a patient treated for miliary TB with central nervous system involvement. During steady-state conditions, the MFX concentration in CSF increased with the dose but not proportionally. The higher drug concentrations in plasma obtained with $800 \mathrm{mg}$ of MFX corresponded to a lower CSF/plasma concentration ratio, possibly reflecting the effect of an induced active transport mechanism resulting in a lower $\mathrm{AUC}_{\mathrm{CSF}} / \mathrm{AUC}_{\text {plasma }}$ ratio at the higher MFX dosage.

The MFX concentrations were considerably lower than those observed in healthy volunteers. Interaction with RIF (4, 13) may partly explain this deviation. The drug interaction between RIF and MFX generally results in a nearly $30 \%$ reduction in the AUC, but in this case a $70 \%$ AUC reduction was observed (10). This additional AUC reduction could result from an increased volume of distribution during inflammatory status. Also, the half-life of MFX (5 h) differed considerably from that in healthy volunteers $(15 \mathrm{~h})$, although it did resemble the half-life of MFX in combination with RIF (13). The shortened half-life may be explained by the interaction with RIF, resulting in increased drug metabolism by enzyme induction.

High-dose MFX treatment was found to be safe in our patient. ECG monitoring did not reveal QT prolongation, and renal and liver functions were not altered. As side effects are often dose and concentration dependent, the low plasma drug levels may explain the absence of side effects.

In our patient with nonactive tuberculous meningitis, adequate MFX concentrations were achieved in CSF, as well as in plasma, with an $\mathrm{AUC}_{\mathrm{CSF}} / \mathrm{AUC}_{\text {plasma }}$ ratio of 0.74 to 0.94 . Monitoring of MFX concentrations may be warranted in patients with interacting comedication or a presumed inadequate AUC/ MIC ratio, based on a high MIC. Our observations suggest that MFX can be used in the treatment of TB with central nervous system involvement if standard treatment is insufficient or poorly tolerated. Still, therapeutic use should be confirmed in a larger population.

We thank Bayer for assistance in analyzing the plasma and CSF samples.

\section{REFERENCES}

1. Blumberg, H. M., W. J. Burman, R. E. Chaisson, C. L. Daley, S. C. Etkind, L. N. Friedman, P. Fujiwara, M. Grzemska, P. C. Hopewell, M. D. Iseman, R. M. Jasmer, V. Koppaka, R. I. Menzies, R. J. O'Brien, R. R. Reves, L. B. Reichman, P. M. Simone, J. R. Starke, and A. A. Vernon. 2003. American Thoracic Society/Centers for Disease Control and Prevention/Infectious Diseases Society of America: treatment of tuberculosis. Am. J. Respir. Crit. Care Med. 167:603-662.

2. Burman, W. J., S. Goldberg, J. L. Johnson, G. Muzanye, M. Engle, A. W. Mosher, S. Choudhri, C. L. Daley, S. S. Munsiff, Z. Zhao, A. Vernon, and R. E. Chaisson. 2006. Moxifloxacin versus ethambutol in the first 2 months of treatment for pulmonary tuberculosis. Am. J. Respir. Crit. Care Med. 174:331-338.

3. Codecasa, L. R., G. Ferrara, M. Ferrarese, M. A. Morandi, V. Penati, C. Lacchini, P. Vaccarino, and G. B. Migliori. 2006. Long-term moxifloxacin in complicated tuberculosis patients with adverse reactions or resistance to first line drugs. Respir. Med. 100:1566-1572.

4. Nijland, H. M. J., R. Ruslami, A. J. Suroto, D. M. Burger, B. Alisjahbana, R. van Crevel, and R. E. Aarnoutse. 2007. Rifampicin reduces plasma concentrations of moxifloxacin in patients with tuberculosis. Clin. Infect. Dis. 45: 1001-1007.

5. Ostergaard, C., T. K. Sorensen, J. D. Knudsen, and N. Frimodt-Moller. 1998. Evaluation of moxifloxacin, a new 8-methoxyquinolone, for treatment of meningitis caused by a penicillin-resistant pneumococcus in rabbits. Antimicrob. Agents Chemother. 42:1706-1712.

6. Rodríguez, J. C., M. Ruiz, M. Lopez, and G. Royo. 2002. In vitro activity of moxifloxacin, levofloxacin, gatifloxacin and linezolid against Mycobacterium tuberculosis. Int. J. Antimicrob. Agents 20:464-467.

7. Rodriguez-Cerrato, V., C. C. McCoig, I. C. Michelow, F. Ghaffar, H. S. Jafri, R. D. Hardy, C. Patel, K. Olsen, and G. H. McCracken, Jr. 2001. Pharmacodynamics and bactericidal activity of moxifloxacin in experimental Escherichia coli meningitis. Antimicrob. Agents Chemother. 45:3092-3097.

8. Shandil, R. K., R. Jayaram, P. Kaur, S. Gaonkar, B. L. Suresh, B. N. Mahesh, R. Jayashree, V. Nandi, S. Bharath, and V. Balasubramanian. 2007. Moxifloxacin, ofloxacin, sparfloxacin, and ciprofloxacin against Mycobacterium tuberculosis: evaluation of in vitro and pharmacodynamic indices that best predict in vivo efficacy. Antimicrob. Agents Chemother. 51:576-582.

9. Stass, H., and A. Dalhoff. 1997. Determination of BAY 12-8039, a new 8-methoxyquinolone, in human body fluids by high-performance liquid chromatography with fluorescence detection using on-column focusing. J. Chromatogr. B Biomed. Sci. Appl. 702:163-174. 
10. Stass, H., D. Kubitza, and U. Schuhly. 2001. Pharmacokinetics, safety and tolerability of moxifloxacin, a novel 8-methoxyfluoroquinolone, after repeated oral administration. Clin. Pharmacokinet. 40(Suppl. 1):1-9.

11. Valerio, G., P. Bracciale, V. Manisco, M. Quitadamo, G. Legari, and S. Bellanova. 2003. Long-term tolerance and effectiveness of moxifloxacin therapy for tuberculosis: preliminary results. J. Chemother. 15:66-70.

12. van Klingeren, B., M. Dessens-Kroon, T. van der Laan, K. Kremer, and D. van Soolingen. 2007. Drug susceptibility testing of Mycobacterium tubercu- losis complex by use of a high-throughput, reproducible, absolute concentration method. J. Clin. Microbiol. 45:2662-2668.

13. Weiner, M., W. Burman, C. C. Luo, C. A. Peloquin, M. Engle, S. Goldberg, V. Agarwal, and A. Vernon. 2007. Effects of rifampin and multidrug resistance gene polymorphism on concentrations of moxifloxacin. Antimicrob. Agents Chemother. 51:2861-2866.

14. Yoshimatsu, T., E. Nuermberger, S. Tyagi, R. Chaisson, W. Bishai, and J. Grosset. 2002. Bactericidal activity of increasing daily and weekly doses of moxifloxacin in murine tuberculosis. Antimicrob. Agents Chemother. 46:1875-1879.

\section{J. W. C. Alffenaar*}

Department of Hospital and Clinical Pharmacy

University Medical Center Groningen

University of Groningen

P.O. Box 30.001

9700 RB Groningen, The Netherlands

\section{P. M. de Vries}

G. J. Luijckx

Department of Neurology

University Medical Center Groningen

University of Groningen

Groningen, The Netherlands

\section{D. van Soolingen}

National Mycobacteria Reference Laboratory

National Institute of Public Health and the Environment

Bilthoven, The Netherlands

\section{T. S. van der Werf}

Departments of Internal Medicine and of Pulmonary Diseases \& Tuberculosis

University Medical Center Groningen

University of Groningen

Groningen, The Netherlands

\section{R. van Altena}

Tuberculosis Centre Beatrixoord

University Medical Center Groningen

University of Groningen

Haren, The Netherlands

*Phone: 31 (0)50 3614070

Fax: 31 (0)50 3614087

E-mail: j.w.c.alffenaar@apoth.umcg.nl

${ }^{\nabla}$ Published ahead of print on 24 March 2008. 